\title{
Original
}

\section{Neutron Emission from Palladium Point Electrode in Pressurized Deuterium Gas under DC Voltage Application}

\author{
by \\ Hiroshi $_{\text {AMADA }^{\dagger} \text { and Tamiya FuJIWARA }}{ }^{\dagger}$
}

\begin{abstract}
The nuclear reaction in palladium electrode under highly non-uniform electric field has been studied using a neutron counting system. Excess neutron counts were observed with deuterium loaded palladium point electrodes in deuterium gas atmosphere in 9 out of 24 runs after activation by flashover between electrodes during DC high voltage application. Similar neutron burst took place without the activation under a DC glow discharge condition in 2 runs out of 37 runs. The tip surface of these two electrodes after the burst was observed to be covered by a large amount of carbon using X-ray photoelectron spectroscopy. Several craters of about $10 \mu \mathrm{m}$ in diameter were formed on the tip surface of one of the two electrodes.
\end{abstract}

Key Words : Palladium, Deuterium Gas, Neutron Emission, Nuclear Fusion, Cold Fusion, Non-uniform Field

\section{Introduction}

Since the cold fusion was claimed to occur ${ }^{1,2)}$ in palladium (Pd) charged during electrolysis of heavy water $\left(\mathrm{D}_{2} \mathrm{O}\right)$, a considerable number of research groups have investigated the nuclear reaction in solid and many positive results have been obtained in these nine years. In one of these studies, De Ninno et al. have found a simple method of the nuclear reaction without the process of electrolysis, that is , they have observed a spontaneous neutron emission from deuterium $\left(\mathrm{D}_{2}\right)$ loaded titanium powder during the process of cooling with liquid nitrogen and heating ${ }^{3)}$. Wada et al. have also reported a spontaneous neutron emission from $\mathrm{Pd}$ electrode in $\mathrm{D}_{2}$ gas during and after activation by flashover between the electrodes ${ }^{4)}$. These studies show that the nuclear reaction takes place at a solid in gas atmosphere as well as in heavy water, even though, the reproducibility of the reaction is low. On the other hand, Takahashi et al. ${ }^{5)}$ have found that current flow condition is a key factor for excess energy production in $\mathrm{D}_{2} \mathrm{O}$ electrolysis. The current density is required to be changed during electrolysis for reaction in $\mathrm{D}_{2}$ loaded electrode. It have been pointed out that current density and loading ratio are another key factors for the start of reaction ${ }^{6}$.

From these point of view, the $d_{2}$ loaded palladium point electrode under highly non-uniform

Received August 31, 1997

$\dagger$ Department of Electrical and Electronic Engineering, Iwate University, Ueda 4-3-5, Morioka 020 Japan 
electric field in $\mathrm{D}_{2}$ gas atmosphere is an attractive arrangement for the study of nuclear reaction. The condition will present high current density at the point under lower voltage application and current fluctuation without change in voltage amplitude. In this study, the nuclear reaction in a $\mathrm{D}_{2}$ loaded Pd point electrode in about 2 atm $\mathrm{D}_{2}$ gas under $\mathrm{DC}$ glow discharge condition has been investigated by neutron detection and X-ray photo-electron spectroscopy (XPS).

\section{Experimental}

A neutron measurement system shown in Fig. 1, is used to detect the excess neutrons from the nuclear reaction in Pd point electrode. The neutron counting system consists of a ${ }^{3} \mathrm{He}$ thermal neutron detector (Reuter-stokes : RS-P4-0806-207), a preamplifier (EG \& G ORTEC : 142PC), an amplifier (EG \& G ORTEC : 590A single channel analyzer), a Timer-and-counter (EG \& G ORTEC : 996). The detector has a sensitive length of $150.9 \mathrm{~mm}$ and outer diameter of $25.4 \mathrm{~mm}$, filled with $4 \mathrm{~atm}$ pressure ${ }^{3} \mathrm{He}$ gas. Signals from the detector are fed to the single channel analyzer through the preamplifier and the amplifier. The counts are stored on a floppy disk using a personal computer. The noise related to high-voltage application is avoided by adjusting the preamplifier gain, the shaping
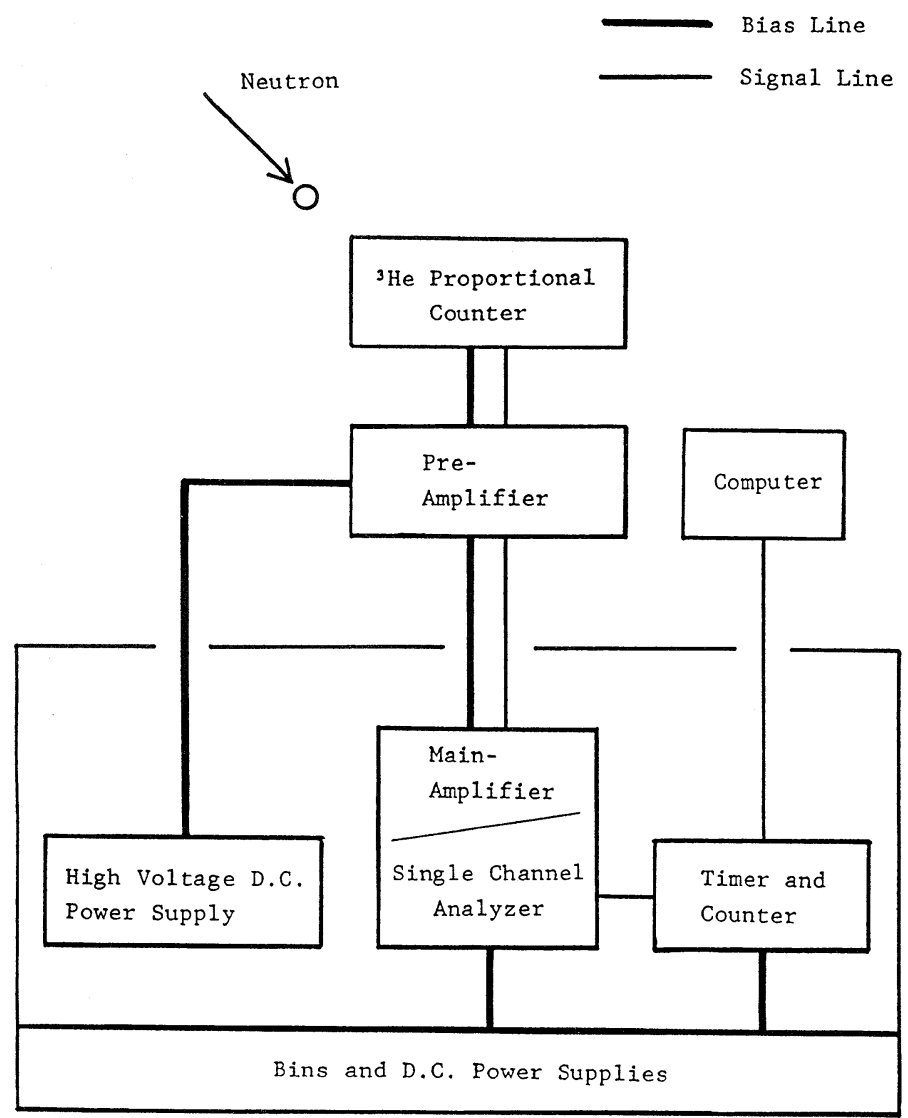

Fig. 1 Schematic diagram of neutron measurement system 

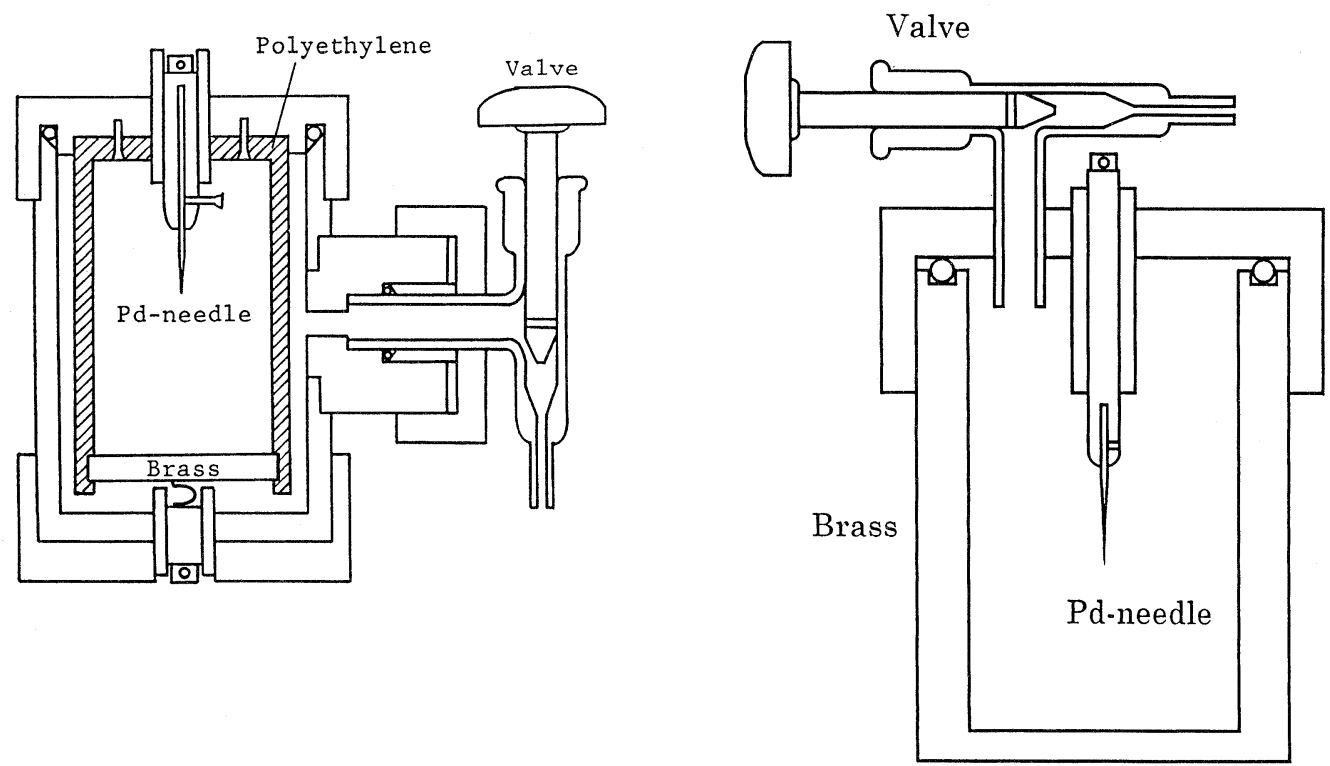

Fig. 2 Tesr cell

$\begin{array}{ll}\text { (a) Cell-A } & \text { (b) Cell-B }\end{array}$

time of the amplifier and the window of the single channel analyzer. The excess neutron counts are recognized by summing up the counts within an interval, in which the counts shows a positive difference from background level.

The two test cells used are shown in Fig. 2 and are assigned to the names "Cell-A" and "Cell-B" . Each cell has a cylindrical shape with volume capacity of $90 \mathrm{~cm}^{3}$ and $110 \mathrm{~cm}^{3}$, respectively. The pointto-plane electrode system with gap spacing $10-22 \mathrm{~mm}$ was employed in the closed cells to obtain highly non-uniform electric field. A Pd wire of $0.5 \mathrm{~mm}$ in diameter was cut to about $30 \mathrm{~mm}$ in length to be a point electrode. After polishing the tip surface of wire with sandpaper, the $\mathrm{Pd}$ point electrode was vacuum annealed at $400-800^{\circ} \mathrm{C}$ for 3 hours under a pressure of about $10^{-4}$ Torr then cooled down to room temperature, followed by loading of $\mathrm{D}_{2}$ gas under 2 atm pressure for 20-40 hours. Next, the Pd point electrode was set into one of the cells, followed by filling the test cell with 2 atm $\mathrm{D}_{2}$ gas. The deuteron to Pd loading ratio was measured to be about 0.6

\subsection{Method for flashover activation using Cell-A}

The neutron moderating system shown in Fig. 3, consists of a polyethylene inner moderator (500mm long, $500 \mathrm{~mm}$ wide and $300 \mathrm{~mm}$ high), $1 \mathrm{~mm}$ thick cadmium (Cd) sheet reflector and 350 $720 \mathrm{~mm}$ thick outer shielding of saturated boric acid solution in water. The polyethylene inner block, having a center cavity with a cylindrical shape of $140 \mathrm{~mm}$ in diameter and $100 \mathrm{~mm}$ high, enhances the efficiency of the ${ }^{3} \mathrm{He}$ counter by moderating fast neutrons emitted from a test cell. The polyethylene moderator is surrounded with the Cd sheet. The test cell and the detector are positioned inside the center cavity. The measurement system is directly positioned on the ground and is covered with only an upper thin roof.

After positioning the cell and the detector inside the center cavity of the moderating system, a 
DC negative $4.5 \mathrm{kV}$ was applied to the Pd point electrode for 24 hours under $2 \mathrm{~atm} \mathrm{D}_{2}$ gas. Then, the lowest DC flashover voltage of about $10 \mathrm{kV}$ was applied to activate the Pd point electrode. Just after several strokes of flashover, the DC voltage was immediately decreased to a slightly lower level than that for flashover. The neutron measurement started just before the flashover took place. It was observed that the flashover and the glow discharge did not affect the counting characteristics of background. Most of runs were carried out for point anode condition; the point electrode was connected to the ground and the DC negative voltage was applied to the plane electrode. Nickel $(\mathrm{Ni})$ and tungsten $(\mathrm{W})$ wires of similar size were used as reference electrodes under the same voltage application with the aim of observing the change in neutron counts. Hydrogen $\left(\mathrm{H}_{2}\right)$ gas and air were also used as loading and environment reference gas.

\subsection{Method for DC glow discharge using Cell-B}

A neutron moderating system, consisting of only the polyethylene block same that shown in Fig. 3 , was used. The system was set in a room at 2 nd floor in a four-storied building. The average background of neutron flax for the system is 25 counts per hour. After positioning the cell and the detector inside the center cavity of the moderating system, positive DC 4 or $8 \mathrm{kV}$ was applied to the Pd point electrode; the plane electrode was connected to the ground. The neutron measurement started just after the voltage application and continued for 24-65 hours.

\section{Result}

The efficiency of the neutron detector was measured to be approximately $1 \%$ using ${ }^{252} \mathrm{Cf}$ source with an intensity of $2.3 \times 10^{4}$ neutrons $\mathrm{s}^{-1}$. The counting rate using the $\mathrm{Cf}$ source is not affected by the distance between the source and the counter, as far as these are in the cavity. A typical time behavior of background neutron with the neutron moderating system for Cell-A is shown in Fig. 4.

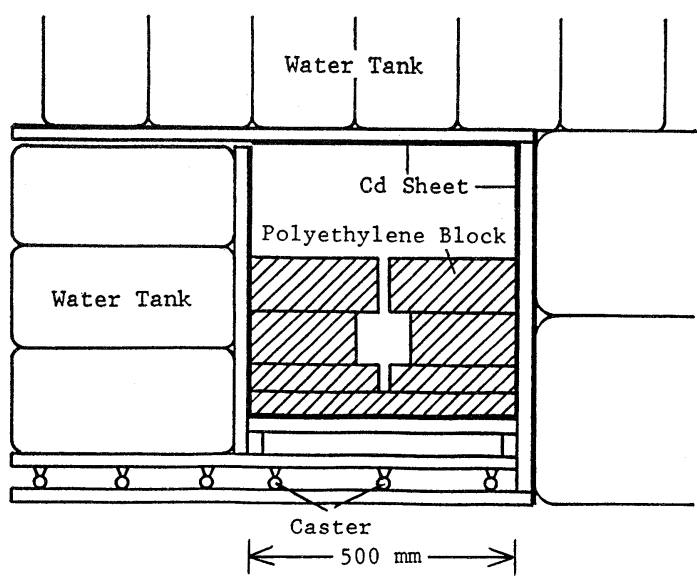

Fig. 3 Neutron moderating system

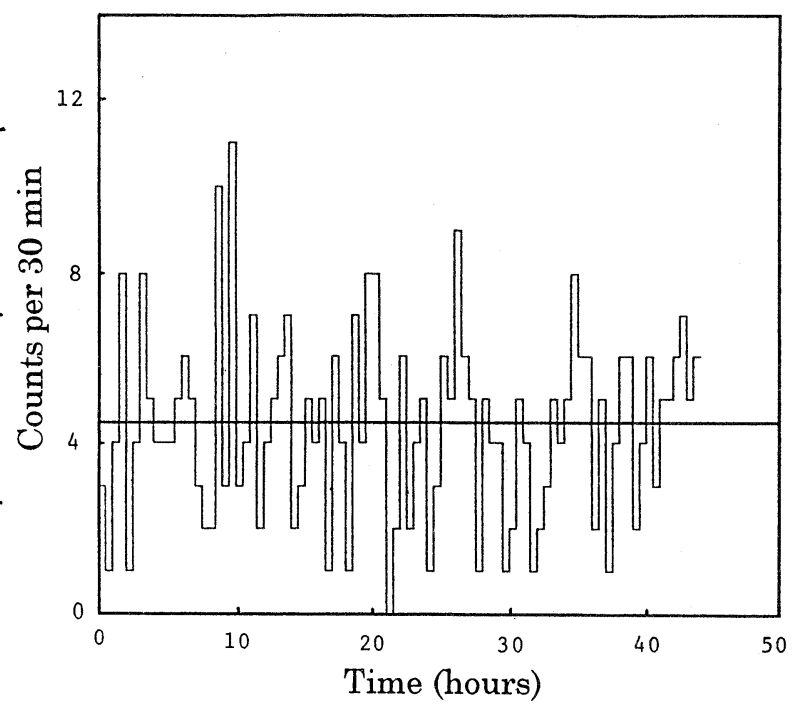

Fig. 4 Time dependence of neutron counts of background 
The average background of neutron flax is 4.3 counts per 30 min ( 8.6 counts per hour) after the adjusting. The horizontal line in the figure gives the average background level. The counting characteristics of background neutrons has been stable over total about 3,500 hours of background measurement period for the past 8 years.

\subsection{Method for flashover activation using Cell-A}

No excess count was usually observed until the activation by the flashover. An initial burst of neutrons was detected under the application of a slightly lower DC voltage than that for flashover just after several strokes of flashover. The burst was observed in 8 out of 24 runs using $\mathrm{D}_{2}$ loaded $\mathrm{Pd}$ point electrodes in $\mathrm{D}_{2}$ gas atmosphere. However, such excess counts was once observed without any flashover under the application of a slightly lower voltage than that for flashover, indicating that the activation by flashover is not always necessary procedure for the neutron emission. These excess counts ceased when the applied voltage was removed. This suggests that the application of DC high voltage is a key factor for the nuclear reaction in this experiment.

Count rate significantly higher than the background level was observed in 6 runs out of these positive 9 runs ; 3 among these remarkable 6 runs were carried out using single Pd electrode. A typical time behavior of neutron emission is shown in Fig. 5 . The count rate is given by the counts summed up within $2 \mathrm{~min}$. The time 0 in the figure shows the time when the last stroke of flashover finished. The average count rate in Fig. 5 is 96.5 counts per 2 min. As compared with this remarkably high count rate, the average background of neutron flax of 0.29 counts per 2 min can be negligible in the figure. Keeping into account the efficiency of the counter, the total number of emitted

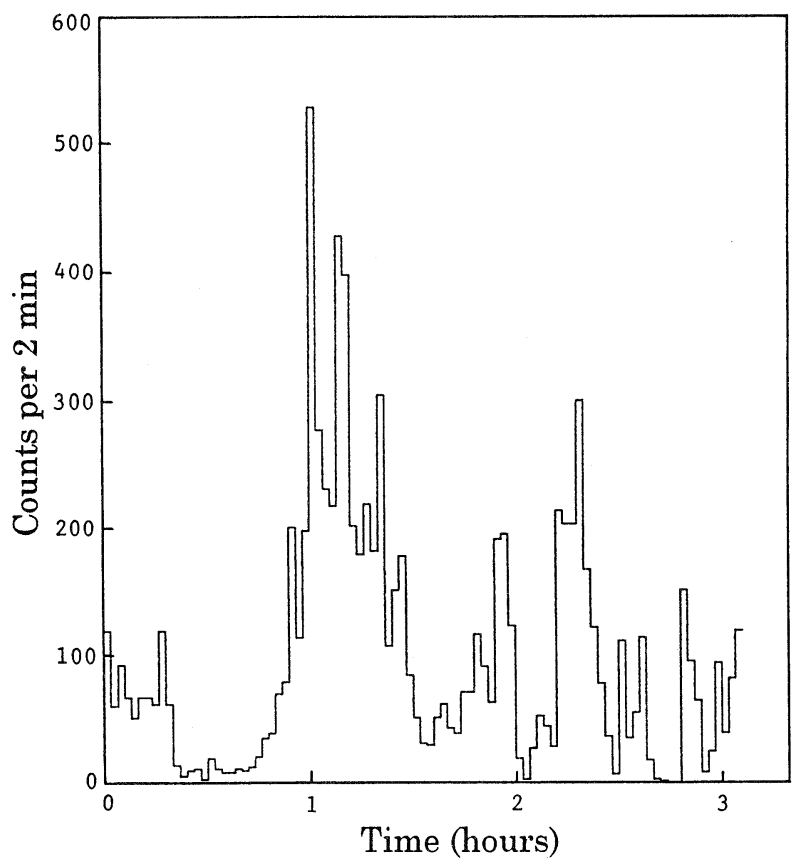

Fig. 5 Time dependence of neutron counts from deuterium-palladium system under positive DC $9.5 \mathrm{kV}$ application after activation by flashover 
neutrons over 3 hours amounts to approximately $1 \times 10^{6}$, assuming that the neutron energy is around $2 \mathrm{MeV}$. The observed highest count rate of 61 counts for $10 \mathrm{sec}$, approximately 2,500 times larger than the background count rate, is equivalent to the emission of $1 \times 10^{5}$ neutrons s $\mathbf{s}^{-1} \mathrm{~cm}^{-3}$ from the Pd point electrode. The DC glow discharge current more than $42 \mu \mathrm{A}$ was measured to flow in the Pd point electrode under the voltage application. The current corresponds to the current density of more than $21 \mathrm{~mA} \cdot \mathrm{cm}^{-2}$ in the Pd point electrode.

In addition, excess neutron emission from $\mathrm{D}_{2}$ loaded $\mathrm{Pd}$ point was also observed in $\mathrm{H}_{2}$ gas atmosphere and in air, while these count rates were lower than that in $\mathrm{D}_{2}$ gas atmosphere. To the contrary, no excess count was observed with other reference point electrodes under the similar test condition. It should be noticed that the case of $\mathrm{H}_{2}$ loaded $\mathrm{Pd}$ point electrode in $\mathrm{D}_{2}$ gas atmosphere gave no excess count. This indicates that the reaction did not take place in the environment gas volume but in Pd bulk or on its surface. The number of positive runs to that of total runs with positive point for each condition is compiled into table 1 ; each numerator shows the number of positive

Table 1 The number of positive runs over that of total runs for point anode condition

\begin{tabular}{|c|c|c|c|c|c|c|c|}
\hline & \multicolumn{6}{|c|}{ Point and (loading gas) } \\
\hline & & $\operatorname{Pd}\left(D_{2}\right)$ & $\mathrm{Pd}\left(\mathrm{H}_{2}\right)$ & $\mathrm{Pd}$ & W & $\mathrm{Ni}\left(\mathrm{D}_{2}\right)$ & $\mathrm{Ni}$ \\
\hline \multirow{3}{*}{ 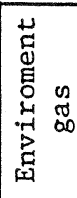 } & $D_{2}$ & $\frac{9}{24}$ & $\frac{0}{6}$ & $\frac{0}{5}$ & $\frac{0}{3}$ & $\frac{0}{8}$ & $\frac{0}{6}$ \\
\hline & $\mathrm{H}_{2}$ & $\frac{2}{4}$ & $\frac{0}{16}$ & $\frac{0}{6}$ & & & \\
\hline & Air & $\frac{1}{2}$ & & & & & \\
\hline
\end{tabular}

runs. All the three positive conditions are encircled with a bold line in the table. The ratio of the positive runs to total runs for each the three conditions give a fairly good reproducibility. Furthermore, A polarity reversed test with $\mathrm{D}_{2}$ loaded $\mathrm{Pd}$ point electrode in $\mathrm{D}_{2}$ gas gave 64 counts in $3 \mathrm{~min}$, suggests that point polarity is not essential factor for the nuclear reaction.

\subsection{Method for DC glow discharge using Cell-B}

The neutron burst was observed in 2 out of 37 runs. The electrode used in the positive 2 runs are assigned to the names "Electrode-A" and "Electrode-B" . The burst from Electrode-A started at 6 hours $48 \mathrm{~min}$ after the beginning of the $8 \mathrm{kV}$ application and continued 3 hours $50 \mathrm{~min}$. No excess count was observed for 30 hours after the burst ceased. The highest count rate of 180 in 3 min, observed just before the burst ceased, is 140 times larger than that of the background neutron. The number of total neutron counted was about 900 .

In the case of Electrode-B, the neutron burst was started just after the $4 \mathrm{kV}$ application and continued $15 \mathrm{~min}$. The highest count rate of about 1,500 in $10 \mathrm{sec}$ is approximately 2,000 times larger than that of background neutron. Next burst started about 5 hours after the first burst and continued $5 \mathrm{~min}$. No excess neutrons were observed for 65 hours after the second burst ceased. The time behavior of the second burst is shown in Fig. 6. The highest count rate of 2,700 in 5 sec was 9 $\times 10^{4}$ times larger than that of the background neutron. The number of neutrons counted during the 2 bursts amounted to $3 \times 10^{4}$. The total number of excess neutrons is estimated to be 35 times 


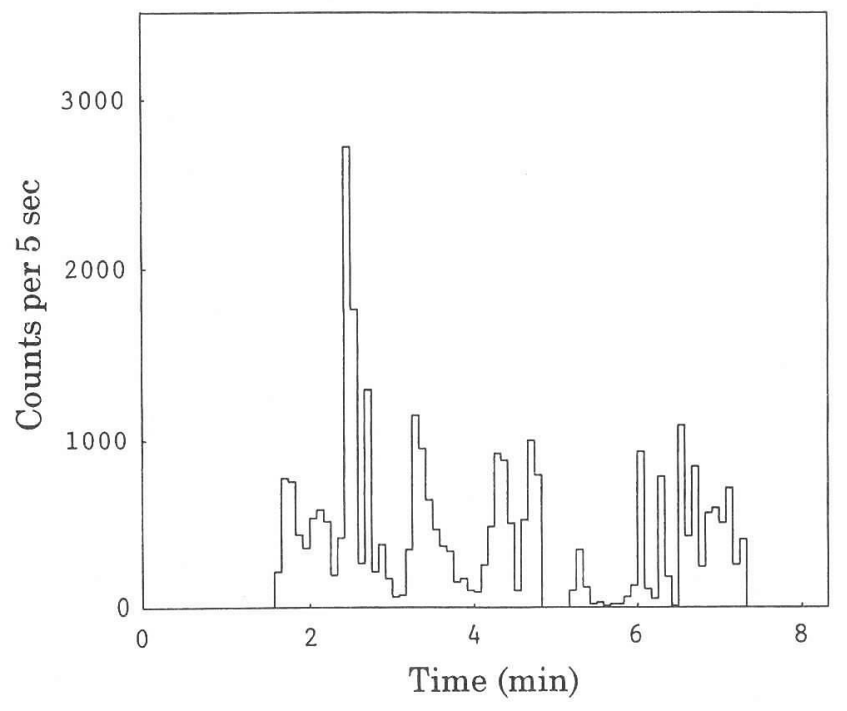

Fig. 6 Time dependence of neutron counts from deuterium-palladium system under positive $\mathrm{DC} \quad 4 \mathrm{kV}$ application without flashover

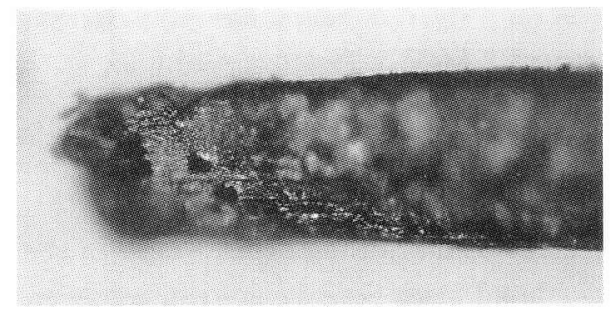

(a)

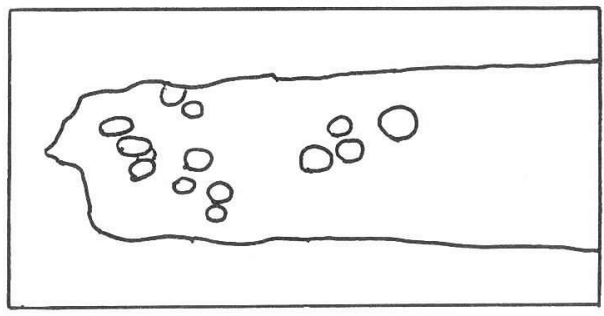

(b)

Fig. 7 (a) Microscopic view of the tip of Electrode-A (b) The sketch of Fig. 7 (a)

larger than that for the Electrode-A, even though the neutron emission period was shorter than that for the Electrode-A.

The electrode after the voltage application were viewed by an optical microscope. The tip surfaces of two positive electrodes were found to be covered with black deposit. The microscopic view of the tip of Electrode-A and its sketch are shown in Fig. 7 (a) and 7 (b), respectively. The area covered with black deposit was measured to be more than $0.32 \mathrm{~mm}^{2}$ for Electrode-A. To the contrary, the tip surfaces of negative 35 electrodes were observed to keep its beginning appearance. Of particular interest is that several craters of about $10 \mu \mathrm{m}$ in diameter, shown in Fig. 7 (b), were formed on the side surface near the tip of Electrode-A. Similar phenomenon has reported by Shoulders et al. ${ }^{7}$ in $\mathrm{D}_{2}$ loaded Pd foils; the spark bombarded Pd foils in air with an electron cluster produce nuclear cluster and form craters on the Pd foils.

An XPS of the tip surface of Electrode-A and B after argon ion etching in about $0.5 \mathrm{~nm}$ revealed that the black deposit is carbon. It seems that the carbon atoms were mixed with Pd atoms in the bulk region from the electrode surface to the depth in several atomic layers at least. The carbon impurity both in the Pd electrode and in the environment $\mathrm{D}_{2}$ gas dose not account for the large amount of $\operatorname{carbon}^{8)}$.

\section{Discussion}

In view of the result presented above, current in Pd point electrode seems to have an important role for the neutron emission from the electrode. The current density of the order of $\mathrm{mA} \cdot \mathrm{cm}^{-2}$ due 
to DC high-voltage application would be responsible for the transition of deuteron from octahedral site to other site ${ }^{9)}$ in the $\mathrm{Pd}$ point electrode. The plausible explanation for the neutron burst and carbon production is that the high current density at the point electrode and its fluctuation by glow discharge would stimulate the accumulation of deuterons to induce a fusion in the bulk near surface of $\mathrm{Pd}$ point electrode.

The deuterium to Pd loading ratio of 0.6 is not considered to be enough value for deuterons to occupy all the octahedral sites in the Pd point electrode. This relatively low loading ratio allow us to conceive that high loading ratio is not always necessary but high current density is rather an important factor for the nuclear reaction. The total excess neutrons estimated was considerably few compared with the estimated number of carbon atoms on the surface of Pd point electrode ${ }^{8)}$. This indicates that a large number of helium would be produced from deuterons at the first reaction step, followed by carbon production from the helium.

\section{Conclusion}

Neutron burst of relatively high reproducibility was observed with $\mathrm{D}_{2}$ loaded $\mathrm{Pd}$ point electrode under DC glow discharge condition just after activation by flashover between electrodes. Such burst was also observed without the activation for longer DC glow discharge condition in 2 atm $\mathrm{D}_{2}$ gas. The large amount of carbon was observed at the tip of electrode after the neutron burst, even though the phenomena are rare. The phenomena could be explained in terms of a nuclear reaction at the surface of Pd point electrode.

\section{References}

1) M. Fleischmann and S. Pons: J. Electroanal. Chem. 261 (1989) 301

2) S. E. Jones et al. : Nature 338 (1989) 737

3) A. De Ninno et al. : Europhys. Lett. 9 (1989) 221

4) N. Wada and K. Nishizawa: Jpn. J. Appl. Phys. 28 (1989) L2017

5) A. Takahashi et al. : Proc. 3rd Int. Conf. Cold Fusion, p. 79 (1993)

6) M. C. H. Mckubre et al. : Proc. 6th Int. Conf. Cold Fusion, p. 75 (1996)

7) K. Shoulders et al. : J. New Energy, 1 (1996) 111

8) H. Yamada et al.: J. New Energy, 1 (1996) 55

9) A. Takahashi et al. : Fusion Tech. 27 (1995) 71 